\title{
Application of a New Wearable Augmented Reality Video See-Through Display to Aid Percutaneous Procedures in Spine Surgery
}

\author{
Fabrizio Cutolo $^{1}$, Marina Carbone ${ }^{1}$, Paolo D. Parchi ${ }^{1,2}$, Vincenzo Ferrari ${ }^{1,3}$, \\ Michele Lisanti ${ }^{1,2}$ and Mauro Ferrari ${ }^{1,4}$ \\ ${ }^{1}$ EndoCAS Center, Department of Translational Research and New Technologies in Medicine \\ and Surgery, University of Pisa, Italy. \\ ${ }^{2} 1$ st Orthopaedic Clinic, University of Pisa, Pisa, Italy. \\ ${ }^{3}$ Information Engineering Department, University of Pisa, Italy. \\ ${ }^{4}$ Department of Vascular Surgery, Pisa University Medical School, Pisa, Italy.
}

\begin{abstract}
In mini-invasive surgery, the surgeon operates without a direct visualization of the patient's anatomy. In image-guided surgery, solutions based on augmented reality (AR) represent the most promising ones. The aim of this study was to evaluate the efficacy of a new wearable AR system as aid in the performance of percutaneous procedures in spine surgery. Our solution is based on a video see-through head mounted display (HMD) and it allows the augmentation of video frames acquired by two external cameras with the rendering of patient-specific 3D models obtained elaborating radiological images. We tested the system on an in vitro setup intended to simulate the reaching of a lumbar pedicle. An experienced surgeon performed the percutaneous task wearing the HMD. System accuracy was evaluated through post-operative CT scan, measuring the maximum distance between the planned and obtained trajectories inside the pedicle canal. The mean insertion error was of $1.18+/-0.16 \mathrm{~mm}$.
\end{abstract}

Keywords: Minimally Invasive Surgery, Augmented Reality and Visualization, Computer Assisted Intervention, Interventional Imaging, Spine Surgery.

\section{Introduction}

Minimally invasive surgery techniques allow the performance of complex procedures with minimized incisions, increased accuracy, and reduced trauma to the patient, and have become the gold standard for the treatment of specific pathological conditions in different surgical disciplines, as in general surgery $[1,2]$ and spine surgery [3]. In mini-invasive surgery the surgeon performs the procedure under medical imaging guidance (endoscope, X-Ray, US, etc.) and he/she is often forced to work 
with an unnatural and restricted view of the surgical field and of the patient's anato$\mathrm{my}$, with the additional information visualized on an external monitor.

In orthopaedic surgery, Vertebroplasty (VP) and Kyphoplasty (KP) are percutaneous procedures widely used for the treatment of osteoporotic or pathologic fractures. Compared to open procedures they bring several advantages as less bleeding, less pain, less risk of infection, small skin incision, early recovery and shorter hospital stays [4-6].

Vertebral augmentation procedures (VAPs) include three main steps: localization of the correct vertebral level; instrumentation of the vertebral body, either transpedicularly or extrapedicularly (cannula insertion); injection of the cement [7]. Fluoroscopic Guidance (FG) is essential in each step, and a substantial exposure to ionizing radiation for surgeon, patient, and medical staff is unavoidable $[8,9]$.

Furthermore, FG provides only bi-dimensional projective images, thus forcing the surgeon to carry out complex processes of mental elaboration and hand-eye coordination. Technological advances in medical imaging technologies (CT, MRI, 3D Ultrasound) have helped to overcome some of the shortcomings and limits hindering the mini-invasive procedures by providing physicians with increasingly detailed information on the anatomy and physiology of the patient. Several research activities have been proposed to move towards the development of surgical navigation systems that could aid the physician throughout all the phases of the procedure.

Surgical navigation systems have been proposed also for VAP, based either on direct intraoperative $3 \mathrm{D}$ imaging and a navigation software $[10,11]$ or on Augmented Reality (AR) strategies [12-14]. In a recent work by Sembrano J. et al. [15], the authors compared navigated (through Stealth computer navigation system by Medtronic) and non-navigated needle placement in 30 balloon kyphoplasty procedures. Their results proved the efficacy of navigated VAP in reducing needle malposition rate, but they were not conclusive in terms of cement leakage rates and clinical benefit for both patient and clinical staff (e.g. radiation exposure to the surgeon and patient). The authors claimed that further studies are still needed to conclusively prove the efficacy of the well-established methods for surgical navigation also in VAPs. On this point, the AR-based approaches may represent a promising breakthrough solution for allowing the smooth integration of surgical navigation into the VAP workflow.

AR-based image guided surgery allows the contextual enrichment of the real view of the surgical scene with a virtual content consisting in specific 3D models of the patient's anatomy extracted from medical dataset [16-18].

This paper aims to propose the use of a new wearable AR video see-through navigation system as an aid in the placement of a working cannula through transpedicular approach into the vertebral body, without the aid of X-ray imaging. The aim of the study is to preliminarily test the accuracy of the system in aiding the reaching of lumbar pedicles, as in a VAP, on a newly designed patient-specific spine phantom. Special focus has been devoted to the evaluation of the ergonomics and the effectiveness of the augmented information to be proposed to the operating surgeon throughout the procedure. 


\section{Material and Methods}

In this section, we shall provide a detailed description of the experimental set-up comprising the HMD system and the patient-specific spine phantom that was designed as testing platform for our AR-based surgical navigation system. Further, we also briefly outline the video see-through paradigm implemented for solving the image-to-patient registration problem.

\subsection{AR navigation system}

Our custom-made stereoscopic video see-through HMDs comprises the following two major components (Fig. 1) [19-21]: a commercial 3D visor and a pair of external USB cameras. The commercial 3D visor is a Sony HMZ-T2, provided with dual 720p OLED panels and a horizontal field of view of $45^{\circ}$. The 2 external USB cameras (uEye XS by IDS) are equipped with a 5 Megapixel CMOS sensor (pixel size of 1.4 $\mu \mathrm{m})$ achieving a frame rate of 15 fps at $1280 \times 720$ resolution. The two external cameras are mounted on the visor aligned with the user's eyes as to provide a quasiorthoscopic view of the surgical scene mediated by the visor (in a video see-through fashion). The AR application was implemented in custom-made software library built in $\mathrm{C}++$ on the top of the multipurpose EndoCAS Navigator Platform modules [22].

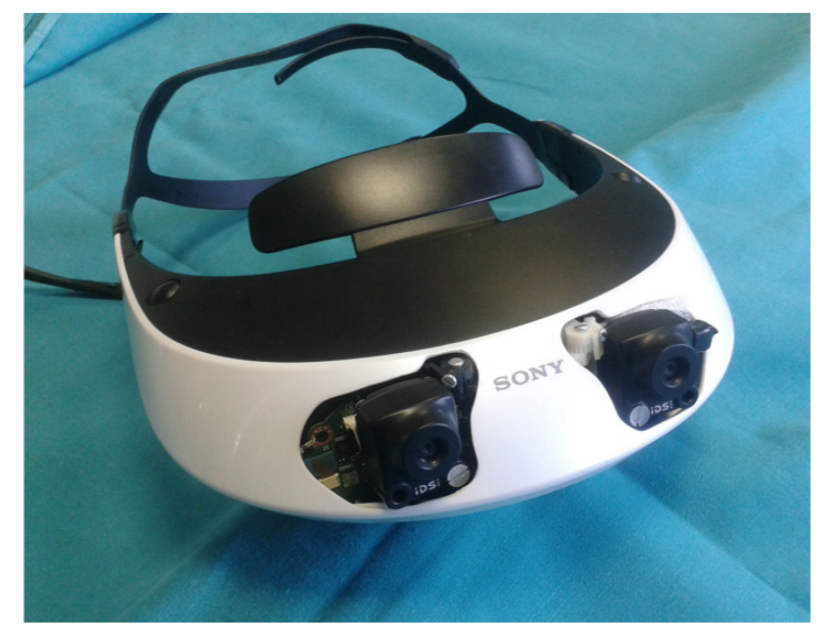

Fig. 1. Our custom-made stereoscopic video see-through HMD based on a commercial 3D visor by Sony (Sony HMZ-T2).

The management of the virtual 3D scene was carried out through the open-source software framework OpenSG 1.8 (www.opensg.org), while regarding the machine vision routines, needed for implementing the video-based tracking method, we adopted Halcon 7.1 software library developed by MVTec®. 
The whole system ran on a gaming laptop Alienware ${ }^{\circledR}$ M14 provided with an Intel Core i7-4700@2.4 GHz quad core processor and 8 GB RAM. The graphics card is a $1 \mathrm{~GB}$ nVidia ${ }^{\circledR}$ GeForce GTX 765M.

Here is a functional and logical overview of the video see-through paradigm underpinning our AR mechanism: the two external cameras grab video frames of the real scene; the video frames are screened as backgrounds onto the corresponding display of the visor; the software application elaborates the grabbed video frames to perform the real-time registration of the virtual content, defined during the surgical planning, to the underlying real scene (Fig. 2).

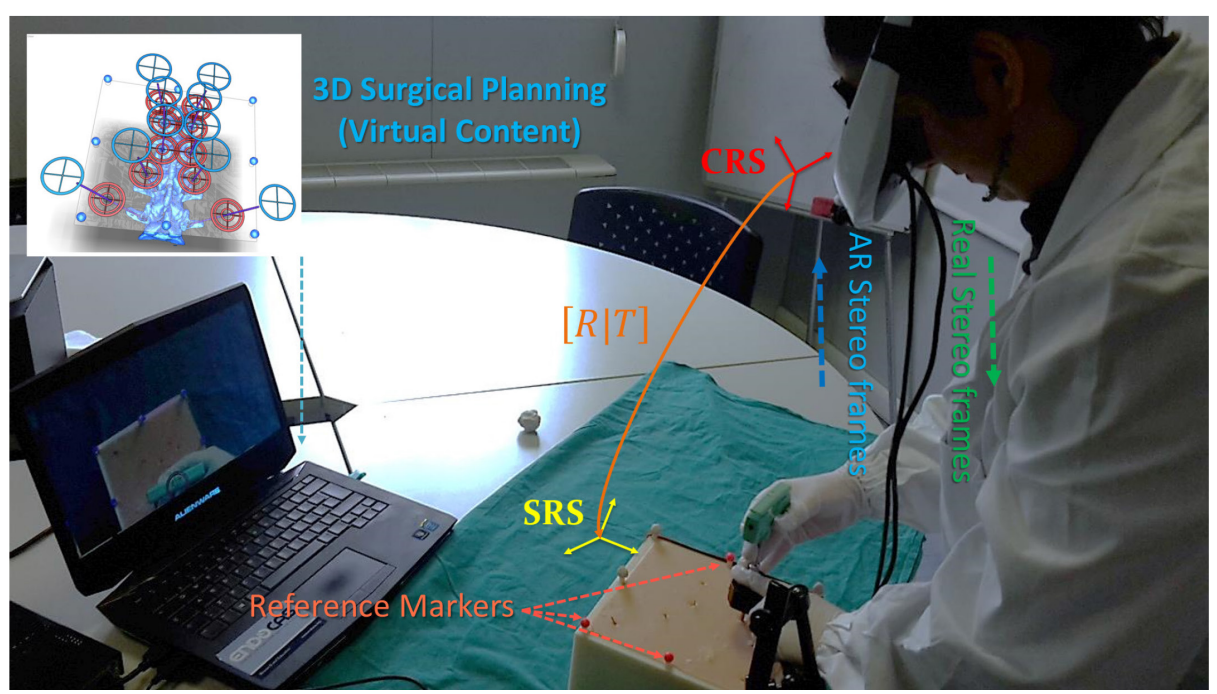

Fig. 2. Video see-through paradigm of the stereoscopic HMD. The software application merges the $3 \mathrm{D}$ surgical planning (virtual content) with the stereoscopic views of the surgical scene (real stereo frames) grabbed by the stereo rig. The AR stereo frames are sent to the two internal monitors of the visor. Alignment between real and virtual information is obtained by a tracking modality that relies on the localization of three reference markers rigidly constrained to the spine phantom and whose position in the virtual scene (SRS) is recorded during surgical planning.

The accurate patient-to-image registration is the fundamental prerequisite for yielding geometric coherence in the AR view of the surgical scene. To fulfill this condition the virtual content is to be observed by a couple of virtual viewpoints (virtual cameras) whose processes of image formation must mimic those of the real cameras in terms of intrinsic and extrinsic parameters. To this end, the intrinsic and extrinsic parameters of the pair of virtual stereo cameras are determined offline through a standard calibration routine [23].

Further, to perform a real time registration of the virtual content to the real scene, our system exploits a video-based tracking modality that relies on the localization of at least three physical markers rigidly constrained to the patient and whose position in the virtual scene (i.e. scene reference system or SRS) is recorded during planning. 
The video-based tracking algorithm computes in real time the rigid transformation encapsulated by matrix $[R \mid T]$, between the camera reference system (CRS) and the SRS [19].

The key characteristic of the implemented strategy for registering the preoperative planning to the live views of the surgical scene is that it does not rely on the adoption of a bulky external tracker. Standard surgical navigation systems, featuring the use of external infrared trackers, may in fact introduce unwanted line-of-sight constraints into the operating room as well as add error-prone technical complexity to the surgical workflow [24]. Our video-based algorithm provides sub-pixel fiducial registration accuracy on the image plane.

\subsection{Spine Phantom}

The accuracy and ergonomics of our AR-based surgical navigation system has been tested on an experimental setup that simulates the reaching of lumbar pedicles as in a standard VAP.

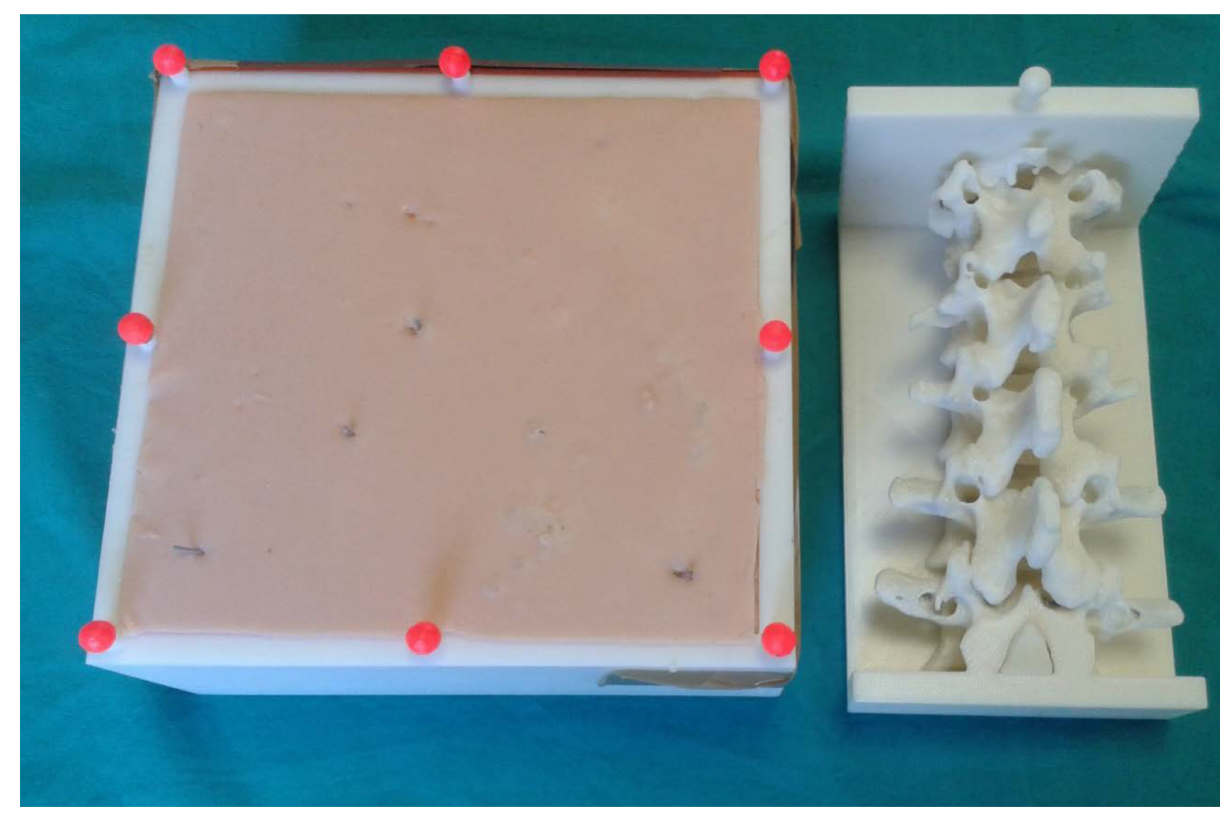

Fig. 3. Model of the lumbar spine with artificially created canals along the pedicles. On the left the ABS replica of the patient-specific phantom was filled with polyurethane foam and covered with a skin-like layer of silicone rubber. On the right the "naked" replica of the spine is shown.

An acrylonitrile butadiene styrene (ABS) replica of a patient-specific phantom comprising the lumbar spine (L1-L5) was created. At first, the 3D model of the lumbar spine was obtained from the segmentation of a preoperative computed tomography (CT) dataset: the DICOM files were segmented using a semi-automatic segmen- 
tation tool integrated into the open-source platform Insight Segmentation and Registration Toolkit [25].

The resulting 3D virtual anatomic details of the spine were then imported in a CAD environment (PTC ${ }^{\circ}$ CREO 3.0) to layout the complete phantom. For each of the five vertebrae, the pair of pedicle canals (left and right one) were identified and artificially created in the form of two cylindrical holes with $6 \mathrm{~mm}$ diameter. The 3D model of the spine was incorporated in a square box that was designed to contain the anatomy and for acting as a support for 8 spheres taken as reference markers for the tracker-less registration (Fig. 3). The obtained CAD model, in the form of a STL file, was then printed with a $3 \mathrm{D}$ rapid prototyping machine (Stratasys ${ }^{\circledR}$ Elite Dimension).

A silicone rubber was injected inside each pedicle in order to replicate the different consistence of the pedicle in respect to the simulated cortical bone. The whole model was then filled with polyurethane foam and covered with a skin-like layer of silicone rubber in order to hide the anatomical target, thus for allowing the simulation of a percutaneous intervention[26].

\subsection{Surgical Planning and AR Visualization modality}

The visually processed data of the AR visualization modality [27] were all conceived to aid the surgeon in planning the optimal trajectory for accessing the surgical target. The 3D virtual reconstruction of the lumbar spine, together with the purely geometrical elements useful for elaborating the visualization modality, were individually exported to a 3D graphics-modelling tool (Right Hemisphere ${ }^{\circledR}$ Deep Exploration) to elaborate the surgical planning.
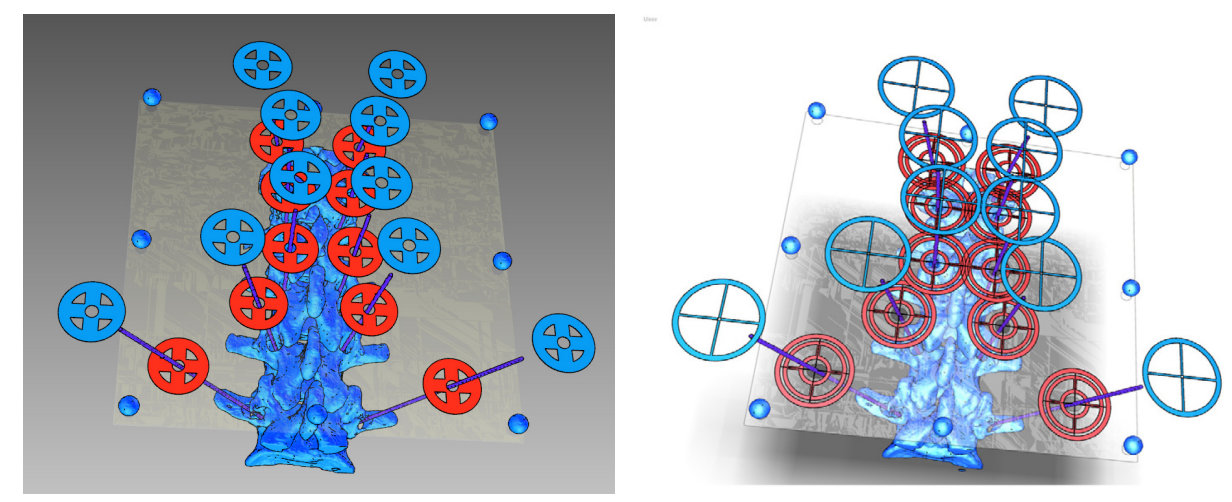

Fig. 4. Complete virtual surgical planning. The figure on the right comprises a more elaborated rendering of the viewfinders, allowing a more accurate definition of the planned trajectory.

Several tests were conducted to evaluate the most ergonomic AR visualization modality in function of the task to be accomplished. In this phase, experienced and 
young surgeons were asked to perform the percutaneous task wearing the HMD with the AR guide running (Fig. 4).

As in previous studies [19, 28-30], also in this specific task, the traditional AR interaction technique, featuring the superimposition of a semi-transparent virtual replica of the spine, did not prove to be very effective in aiding the surgeon in targeting the pedicle canal. This is due to the surgeon's limited perception of the relative distances of objects within the AR scene and to the presence of unnatural occlusions between real and virtual structures. Further, the presentation of a too detailed and complex virtual content, proved to confound the surgeon instead of being of assistance. A major issue in the designing of AR-based surgical navigation system is related to the need of providing consistent visual cues for correct perception of depth and spatial relations in the augmented scene [31].

In this regard, the most ergonomic AR visualization modality has proven to be a view indicating the ideal trajectory for targeting each pedicle through a pair of virtual viewfinders: the first at the level of the skin, the second at the level of the bottom of the trocar. The first viewfinder indicates the ideal insertion point for the surgical tool, whereas the second viewfinder defines, once coaxially aligned with the first one, the optimal trajectory of trocar insertion. With such AR guide, the surgeon must simply align the tip of the needle to the center of the first viewfinder on the patient's skin (hence managing 2 positional degrees of freedom).

The second viewfinder is then used by the surgeon to pivoting the trocar around the entry point so that it can be aligned to the planned insertion direction into the pedicle (managing 2 rotational degrees of freedom) (Fig. 5).
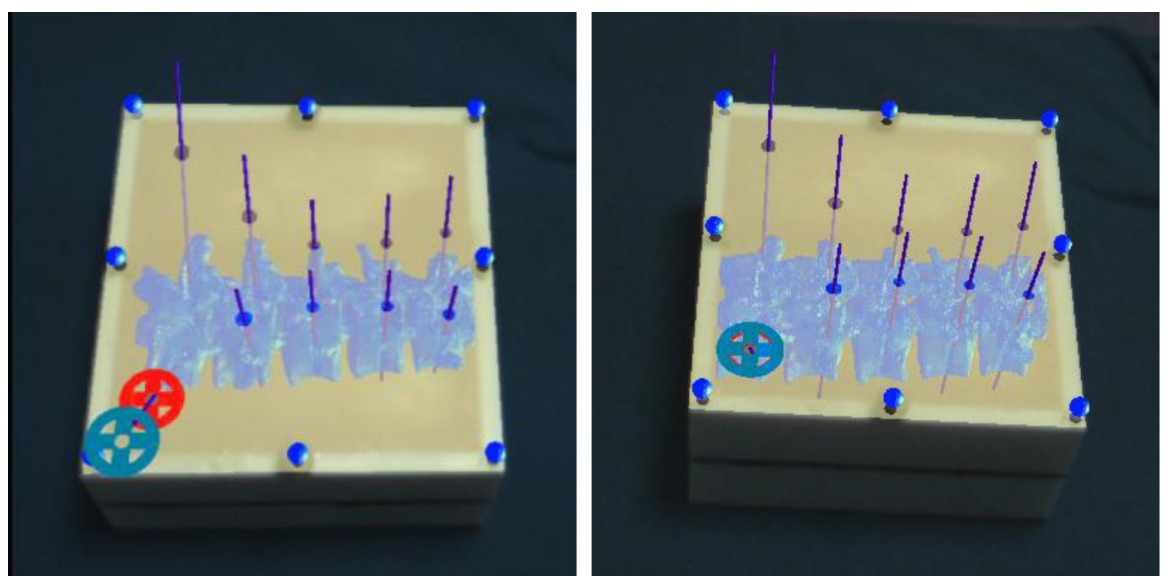

Fig. 5. AR Visualization with the rendering of the spine, the skin, the ideal trajectories, and a pair of viewfinders defining an optimal trajectory of insertion. 


\subsection{Evaluation Study}

In the preliminary evaluation tests, an experienced surgeon performed 4 trial sessions. Each test session consisted in the insertion of 10 Kirshner-Wires (K-Wires) in the pair of pedicles of the 5 lumbar vertebrae included in the phantom. The tests were performed with the aid of the stereoscopic HMD and using the above described AR Visualization modality. The surgeon repeated the test 4 times for a total of 40 percutaneous insertions.

These preliminary tests were aimed at assessing the efficacy of the AR-based guide in terms of accuracy in needle insertion. In this in vitro study, we have not evaluated the registration accuracy, since the in vitro setup did not give rise to any issue related to a preoperative registration procedure: the fiducial markers were rigidly constrained to the ABS box used as support for the replica of the spine.

The surgeon, once planned the ideal trajectory, started the trial whilst wearing the HMD device. The first viewfinder on the external surface of the phantom (i.e. the skin) helped the surgeon finding the insertion point; the second viewfinder, at the bottom of the trocar, was intended to aid the surgeon in defining the optimal trajectory of insertion. Once the surgeon considered the trajectory as satisfying, the K-wire could be inserted.

For each session, system accuracy was evaluated by using post-operative CT scans of the model of the lumbar spine with the 10 needles inserted (Fig. 6). After registering the preoperative and the postoperative reconstructions of the lumbar spine, three different engineers evaluated insertion errors for each $\mathrm{K}$-wire at the upper and lower surface of each pedicle canal by comparing the planned trajectories (green lines in Fig. 6) with the real ones (red lines in Fig. 6). Likewise, the angular insertion error in the axial and sagittal plane was measured. Insertion errors were computed by means of the values collected by the three technicians.


Fig. 6. Evaluation of the insertion error between planned trajectories (green lines) and reached trajectories (red lines). The light green cylinders indicate the pedicle canals.

It is important to highlight that, from session 2, a different approach for needle insertion was adopted: a clamping arm was introduced to hold the trocar steady in the 
defined trajectory during the insertion phase. This strategy was adopted following on surgeon's feedback on the difficulty encountered in maintaining the trajectory steady during the needle insertion. By means of the clamping arm, the trocar can in fact be fastened once the planned trajectory is reached, thus allowing a straight trocar insertion. Therefore, the surgical tasks can be summarized in (1) align the tip of the trocar to the center of the first viewfinder and (2) align the trocar bottom to the second viewfinder (Fig. 7).
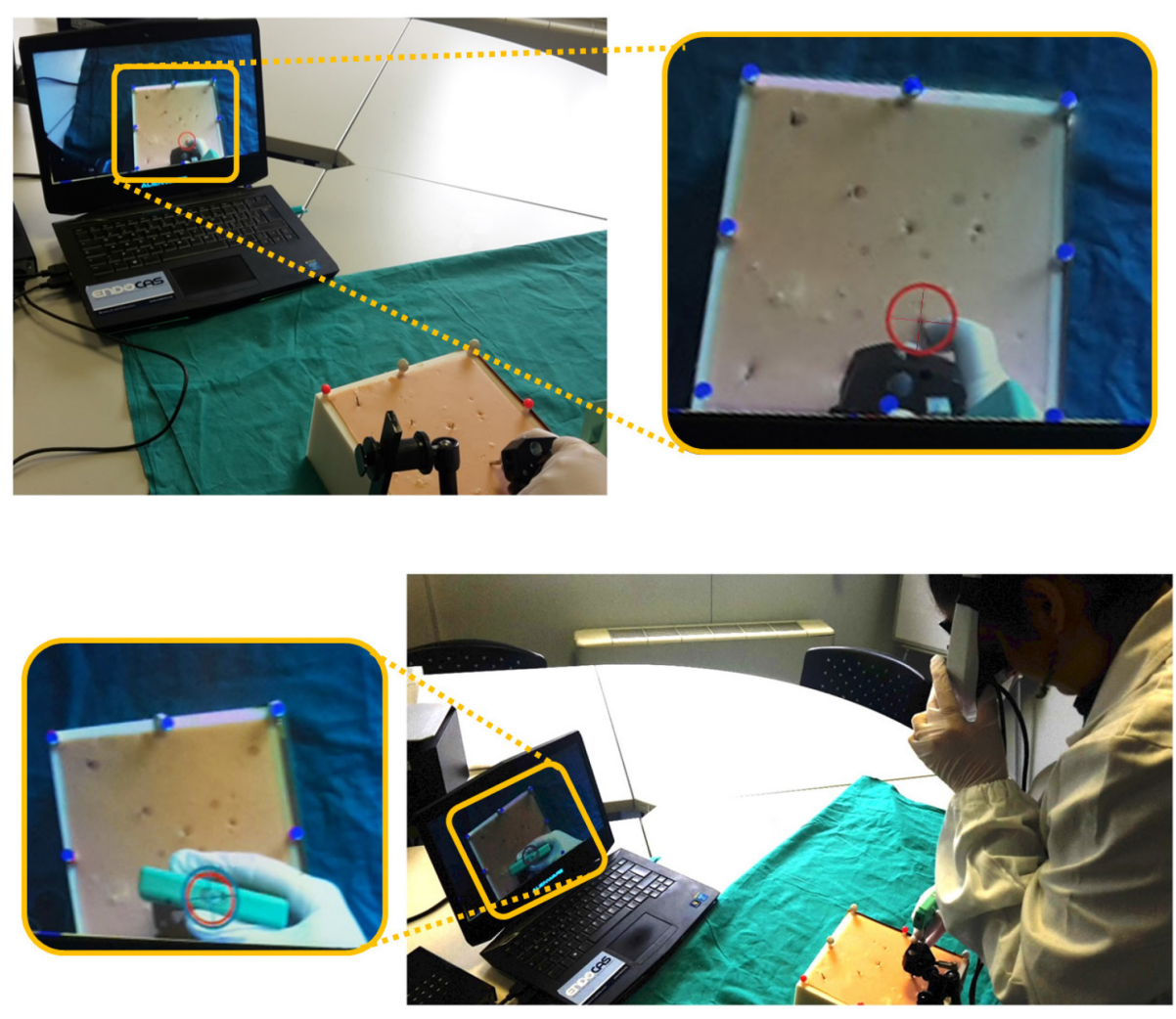

Fig. 7. The AR-aided surgical tasks. Upper row: The surgeon first aligns the tip of the trocar to the center of the red viewfinder he/she sees in the AR scene; in the zoomed image the red viewfinder and the centered tip are shown. Lower row: the surgeon coaxially aligns the handle of the trocar to the two viewfinders (blue and red). The two viewfinders define the ideal trajectory of insertion; in the zoomed image the two viewfinders and the handle of the trocar are shown optimally aligned. 


\section{$3 \quad$ Results}

Results of the tests are reported in Table 1. The results obtained in all the 4 test sessions (10 K-wires implanted for each session) show an overall mean error of 1.18 $\mathrm{mm}$ (range of misplacement: $0.01-2.45 \mathrm{~mm}$ ) with $2 \mathrm{~K}$-wires misplaced (L1Sx and L3sx) in the first session, the one in which the clamping arm was not used.

Table 1. Insertion errors of the needle inside the pedicles. err US: error at the Upper Surface. err LS: error at the Lower Surface. AxA err: Axial Angular error. SagA err: Sagittal Angular Error. Mean errors and Standard Deviation are reported for each session test and globally.

\begin{tabular}{c|ccccc}
\hline & err US [mm] & err LS [mm] & Overall Mean & AxA err $\left[^{\circ}\right]$ & SagA err $\left[^{\circ}\right]$ \\
\hline $\begin{array}{c}\text { Test 1 } \\
\text { (hands free) } \\
\text { Test 2 }\end{array}$ & $1.27 \pm 0.71$ & $1.58 \pm 0.60$ & $\mathbf{1 . 4 2} \pm \mathbf{0 . 6 6}$ & $1.97 \pm 1.53$ & $1.26 \pm 0.95$ \\
& $1.13 \pm 0.50$ & $1.04 \pm 0.36$ & $\mathbf{1 . 0 8} \pm \mathbf{0 . 4 3}$ & $1.20 \pm 0.61$ & $0.75 \pm 0.45$ \\
Test 3 & $1.03 \pm 0.70$ & $1.29 \pm 0.43$ & $\mathbf{1 . 1 6} \pm \mathbf{0 . 5 8}$ & $1.32 \pm 0.89$ & $1.24 \pm 0.54$ \\
Test 4 & $1.03 \pm 0.40$ & $1.11 \pm 0.44$ & $\mathbf{1 . 0 7} \pm \mathbf{0 . 4 1}$ & $1.44 \pm 0.78$ & $1.09 \pm 0.68$ \\
$\begin{array}{c}\text { Overall } \\
\text { MEAN }\end{array}$ & $\mathbf{1 . 1 1} \pm \mathbf{0 . 1 2}$ & $\mathbf{1 . 2 5} \pm \mathbf{0 . 2 4}$ & $\mathbf{1 , 1 8} \pm \mathbf{0 . 1 6}$ & $\mathbf{1 . 4 8} \pm \mathbf{0 . 3 4}$ & $\mathbf{1 . 0 8} \pm \mathbf{0 . 2 3}$ \\
\hline
\end{tabular}

After the introduction of the clamping arm (i.e. in the last 3 test sessions) we recorded a reduction of the overall mean error from 1.42 to $1.1 \mathrm{~mm}$. As for the angular errors, we obtained an average error of $1.48^{\circ}$ (range of angular error: $1.20^{\circ}-1.97^{\circ}$ ) in the axial plane and $1.08^{\circ}$ (range: $0.75-1.26^{\circ}$ ) in the sagittal plane.

The errors reported are encouraging and comparable with those reported in literature. A similar AR-guided approach was described by Abe et al. in 2013 . They presented the results of a study conducted on phantoms and on 5 patients in which they adopted a modified commercial HMD (Epson ${ }^{\circledR}$ Moverio) as an AR-based aid in the definition of the optimal trajectory path towards the pedicle canal. Their system uses a single webcam and a template-based tracking method. Throughout the study, they inserted $40 \mathrm{~K}$-wires in a phantom with two vertebrae with and without the navigation system. Axial and sagittal angular errors of insertion for each K-wire were measured by means of a control CT. The overall average angular error reported was of about $0.75 \pm 0.61^{\circ}$ on the axial-plane and $0.61 \pm 0.70^{\circ}$ in the sagittal-plane. The results highlighted a statistically significant improvement of the accuracy with the guidance system.

Fritz et al. in 2014 reported their experience with a MRI guided approach. In their study, they performed 25 PVP on a cadaver study reporting a final target error of $6.1 \pm 1.9 \mathrm{~mm}$ [14]. Braak et al. in 2013 [10] proposed a Cone Bean CT-based AR guided approach. In that work, the planned trajectory, with a 5-mm safety margin for 
the needle path, is defined directly in the operating room. The planned path is then projected onto the fluoroscopy image producing a highly accurate real-time image of needle positioning and progression toward the target. The authors tested the system on a spine phantom for a total of 44 needles inserted reporting an overall accuracy of $2.61 \pm 1.72 \mathrm{~mm}$.

\section{Conclusion}

There is a growing interest on the use of AR systems as new surgical navigation systems. The introduction of AR in orthopedic surgery, both for training purposes and as surgical navigators, can lead to positive and encouraging results in terms of increased accuracy and reduced trauma to the patient.

Wearable AR systems based on HMDs allows the surgeon to have an ergonomic viewpoint of the surgical field and of the patient's anatomy and reduce the problems related to eye-hand coordination [19].

In this study, we have presented a wearable AR navigation system as aid to percutaneous VAPs. Results of the in vitro tests were encouraging in terms of insertion accuracy, system usability and ergonomics. Quantitative results confirmed the feasibility of this approach: although our testing platform did not comprise any registration phase, our results, in terms of insertion accuracy, were comparable to similar studies from the published literature. The next appropriate steps will be to proceeding to testing on humans to assess, under real clinical conditions, surgical accuracy and real benefits for the patient. To this end, we think that an improvement of the graphical interface and the designing of a robust intraoperative registration procedure will be key steps to be addressed to improve system accuracy and ergonomics.

Acknowledgments. This work was funded by the Italian Ministry of Health grant SThARS (Surgical training in identification and isolation of deformable tubular structures with hybrid Augmented Reality Simulation, 6/11/2014 - 5/11/2017). Grant "Ricerca finalizzata e Giovani Ricercatori 2011-2012" Young Researchers - Italian Ministry of Health.

\section{References}

1. Soper, N.J., Stockmann, P.T., Dunnegan, D.L., Ashley, S.W.: Laparoscopic cholecystectomy. The new 'gold standard'? Archives of surgery 127, 917-921; discussion 921-913 (1992)

2. Legorreta, A.P., Silber, J.H., Costantino, G.N., Kobylinski, R.W., Zatz, S.L.: Increased cholecystectomy rate after the introduction of laparoscopic cholecystectomy. Jama 270, 1429-1432 (1993)

3. Oppenheimer, J.H., DeCastro, I., McDonnell, D.E.: Minimally invasive spine technology and minimally invasive spine surgery: a historical review. Neurosurgical focus 27, E9 (2009) 
4. Deramond, H., Sebert, J.L., Rosat, P., Fardellone, P., Romero, C.A., Berlemont, F.: Destructive spondyloarthropathy in chronic haemodialysis patients. Current data and radiological aspects. Journal of neuroradiology. Journal de neuroradiologie 14, 27-38 (1987)

5. Peh, W.C., Gilula, L.A.: Percutaneous vertebroplasty: indications, contraindications, and technique. The British journal of radiology 76, 69-75 (2003)

6. Garfin, S.R., Yuan, H.A., Reiley, M.A.: New technologies in spine: kyphoplasty and vertebroplasty for the treatment of painful osteoporotic compression fractures. Spine 26, 1511-1515 (2001)

7. Peh, W.C.G., Gilula, L.A.: Percutaneous vertebroplasty: indications, contraindications, and technique. The British Journal of Radiology 76, 69-75 (2003)

8. Ortiz, A.O., Natarajan, V., Gregorius, D.R., Pollack, S.: Significantly reduced radiation exposure to operators during kyphoplasty and vertebroplasty procedures: methods and techniques. AJNR. American journal of neuroradiology 27, 989-994 (2006)

9. Choi, H.C.: Fluoroscopic Radiation Exposure during Percutaneous Kyphoplasty. Journal of Korean Neurosurgical Society 49, 37-42 (2011)

10. Braak, S.J., Zuurmond, K., Aerts, H.C., van Leersum, M., Overtoom, T.T., van Heesewijk, J.P., van Strijen, M.J.: Feasibility study of needle placement in percutaneous vertebroplasty: cone-beam computed tomography guidance versus conventional fluoroscopy. Cardiovascular and interventional radiology 36, 1120-1126 (2013)

11. Hoheisel, M., Skalej, M., Beuing, O., Bill, U., Klingenbeck-Regn, K., Petzold, R., Nagel, M.H.: Kyphoplasty interventions using a navigation system and C-arm CT data: first clinical results. pp. 72580E-72580E-72588. (Year)

12. Bichlmeier, C., Ockert, B., Heining, S.M., Ahmadi, A., Navab, N.: Stepping into the Operating Theater: ARAV - Augmented Reality Aided Vertebroplasty. 7th Ieee International Symposium on Mixed and Augmented Reality 2008, Proceedings 165-166 (2008)

13. Abe, Y., Sato, S., Kato, K., Hyakumachi, T., Yanagibashi, Y., Ito, M., Abumi, K.: A novel 3D guidance system using augmented reality for percutaneous vertebroplasty. J Neurosurg-Spine 19, 492-501 (2013)

14. Fritz, J., P, U.T., Ungi, T., Flammang, A.J., Kathuria, S., Fichtinger, G., Iordachita, II, Carrino, J.A.: MR-guided vertebroplasty with augmented reality image overlay navigation. Cardiovascular and interventional radiology 37, 1589-1596 (2014)

15. Sembrano, J.N., Yson, S.C., Polly, D.W., Jr., Ledonio, C.G., Nuckley, D.J., Santos, E.R.: Comparison of nonnavigated and 3-dimensional image-based computer navigated balloon kyphoplasty. Orthopedics 38, 17-23 (2015)

16. Kersten-Oertel, M., Jannin, P., Collins, D.L.: The state of the art of visualization in mixed reality image guided surgery. Comput Med Imag Grap 37, 98-112 (2013)

17. Cutolo, F., Badiali, G., Ferrari, V.: Human-PnP: Ergonomic AR Interaction Paradigm for Manual Placement of Rigid Bodies. In: Linte, C., Yaniv, Z., Fallavollita, P. (eds.) Augmented Environments for Computer-Assisted Interventions, vol. 9365, pp. 50-60. Springer International Publishing (2015)

18. Sielhorst, T., Feuerstein, M., Navab, N.: Advanced Medical Displays: A Literature Review of Augmented Reality. J Disp Technol 4, 451-467 (2008)

19. Cutolo, F., Parchi, P.D., Ferrari, V.: Video See Through AR Head-Mounted Display for Medical Procedures. Int Sym Mix Augment 393-396 (2014)

20. Ferrari, V., Cutolo, F., Calabro, E.M., Ferrari, M.: HMD Video See Though AR with Unfixed Cameras Vergence. Int Sym Mix Augment 265-266 (2014) 
21. Ferrari, V., Megali, G., Troia, E., Pietrabissa, A., Mosca, F.: A 3-D Mixed-Reality System for Stereoscopic Visualization of Medical Dataset. Ieee T Bio-Med Eng 56, 2627-2633 (2009)

22. Megali, G., Ferrari, V., Freschi, C., Morabito, B., Turini, G., Troia, E., Cappelli, C., Pietrabissa, A., Tonet, O., Cuschieri, A., Dario, P., Mosca, F.: EndoCAS navigator platform: a common platform for computer and robotic assistance in minimally invasive surgery. Int J Med Robot Comp 4, 242-251 (2008)

23. Zhang, Z.Y.: A flexible new technique for camera calibration. Ieee T Pattern Anal 22, $1330-1334$ (2000)

24. Navab, N., Heining, S.M., Traub, J.: Camera Augmented Mobile C-Arm (CAMC): Calibration, Accuracy Study, and Clinical Applications. Ieee T Med Imaging 29, 14121423 (2010)

25. Ferrari, V., Carbone, M., Cappelli, C., Boni, L., Melfi, F., Ferrari, M., Mosca, F., Pietrabissa, A.: Value of multidetector computed tomography image segmentation for preoperative planning in general surgery. Surg Endosc 26, 616-626 (2012)

26. Condino, S., Carbone, M., Ferrari, V., Faggioni, L., Peri, A., Ferrari, M., Mosca, F.: How to build patient-specific synthetic abdominal anatomies. An innovative approach from physical toward hybrid surgical simulators. Int J Med Robot 7, 202-213 (2011)

27. Kersten-Oertel, M., Jannin, P., Collins, D.L.: DVV: A Taxonomy for Mixed Reality Visualization in Image Guided Surgery. Ieee T Vis Comput Gr 18, 332-352 (2012)

28. Badiali, G., Ferrari, V., Cutolo, F., Freschi, C., Caramella, D., Bianchi, A., Marchetti, C.: Augmented reality as an aid in maxillofacial surgery: Validation of a wearable system allowing maxillary repositioning. J Cranio Maxill Surg 42, 1970-1976 (2014)

29. Parrini, S., Cutolo, F., Freschi, C., Ferrari, M., Ferrari, V.: Augmented reality system for freehand guide of magnetic endovascular devices. Conf Proc IEEE Eng Med Biol Soc 2014, 490-493 (2014)

30. Ferrari, V., Viglialoro, R.M., Nicoli, P., Cutolo, F., Condino, S., Carbone, M., Siesto, M., Ferrari, M.: Augmented reality visualization of deformable tubular structures for surgical simulation. Int J Med Robot (2015)

31. Bichlmeier, C., Wimme, F., Heining, S.M., Navab, N.: Contextual Anatomic Mimesis Hybrid In-Situ Visualization Method for Improving Multi-Sensory Depth Perception in Medical Augmented Reality. In: Mixed and Augmented Reality, 2007. ISMAR 2007. 6th IEEE and ACM International Symposium on, pp. 129-138. (Year) 